\title{
Clinical Effect of Integrated Traditional Chinese and Western Medicine on Diabetic Peripheral Neuropathy
}

\author{
Hao Peng1, Yuhong Duan* \\ ${ }^{1}$ Shaanxi University of Chinese Medicine, Xianyang 712046, Shaanxi Province, China \\ ${ }^{2}$ The Affiliated Hospital of Shaanxi University of Chinese Medicine, Xianyang 712000, Shaanxi Province, China \\ *Corresponding author: Yuhong Duan, 56782039@qq.com
}

\begin{abstract}
Objective: Patients with diabetic peripheral neuropathy were treated with integrated traditional Chinese and western medicine, and the final results were observed. Methods: 70 patients with such symptoms were randomly divided into two groups. The number of people is the same, and the treatment methods are different. The control group uses western medicine, while the observation group uses traditional Chinese medicine to evaluate the treatment effect. Results: After treatment, the effective rate of the observation group was $(\mathrm{P}<0.05)$, indicating that the treatment effect was significantly better. Conclusion: Patients with clinical diabetic peripheral neuropathy treated with routine nutrition and nerve repair, and with Chinese medicine, the effect is more satisfactory, and the effect is positive, so as to promote the optimization of their health.
\end{abstract}

Keywords: Combination of traditional Chinese and Western medicine; Clinical treatment; Effect; Diabetic peripheral neuropathy; Observation; Analysis

Publication date: November 2021; Online publication: November 30, 2021

\section{Introduction}

Diabetes is more common, and because of the gradual increase of patients and the gradual extension of the disease, the number of patients with peripheral neuropathy has been improved. It is one of the most common complications of this kind of disease. It is accompanied by a series of abnormal physical sensation, and it can also cause damage ${ }^{[1]}$. For such diseases, in the past, the main treatment scheme was to nourish nerves, repair nerves and control blood glucose, but the final treatment effect was relatively limited ${ }^{[2]}$. It was necessary to receive corresponding treatment for a long time to promote the prognosis of their existing diseases. Therefore, other treatment methods were added to coordinate treatment intervention, which has certain practical value and key advantages ${ }^{[3]}$. After the application of integrated traditional Chinese and western medicine, the existing health status of patients can be changed, which can further promote the improvement of disease problems and actively optimize the symptoms of limb numbness and pain in the early stage. Based on this, this study compares the effects of relevant methods after clinical treatment, and compares and evaluates the application advantages ${ }^{[4]}$.

\section{Materials and methods}

\subsection{General information}

Seventy patients were randomly divided into two groups $(n=35)$. The patients were $34-78$ years old, with an average of $54.32 \pm 11.37$ years. From the onset of the disease to the present 3-11 years, with an average of $7.24 \pm 1.35$ years. At this stage, it is accompanied by limb numbness, abnormal perception, glove and sock like sensation, decreased motor ability and muscle tension, and there are no other skin diseases, 
nervous system diseases, limb vascular diseases and other problems ${ }^{[5]}$. It can cooperate and comply with relevant therapeutic drugs and intervention methods normally, and there are no mental disorders, mood disorders, immune disorders, other major complications or chronic diseases, coagulation disorders, the history of dementia and related diseases. The patients are highly close to each other $(\mathrm{P}>0.05)$, which meets the requirements of the principles and standards of the control trial, and can be compared to evaluate the treatment effect ${ }^{[6]}$.

\subsection{Methods}

Patients in the control group received routine clinical disease treatment, reasonably controlled blood glucose, controlled their primary diseases, kept blood glucose and glycosylated hemoglobin at a normal level, and adjusted blood lipid and blood pressure ${ }^{[7]}$. They increased the use of vitamin B12, mecobalamin and lipoic acid, in which the frequency of VB12 is $0.5 \mathrm{~g}$ once a day, intramuscular injection. Mecobalamin is taken orally, with a frequency of $0.5 \mathrm{mg}$ three times a day. At the same time lipoic acid is used for intravenous drip. 300mg lipoic acid needs to be mixed with $250 \mathrm{ml}$ normal saline for drip once a day. On this basis, the observation group was treated with traditional Chinese medicine. Huangqi Guizhi Wuwu decoction was selected, including Radix Astragali, 35g Caulis Spatholobi, 30g of Flos Lonicerae, 20g of Radix Paeoniae Rubra, 15g of Radix Salviae Miltiorrhizae, Rhizoma Ligustici and mulberry, 10g of Ramulus Cinnamomiand, $5 \mathrm{~g}$ of Fructus Jujubae and 3 pieces of ginger, combined with $6 \mathrm{~g}$ of fried Radix Glycyrrhizae. The decoction was given once a day, $200 \mathrm{ml}$ of its liquid was decocted with water, and then taken after warming.

\subsection{Observation indexes}

The total effective rate of the two groups after treatment was observed and compared. If after clinical treatment, all kinds of clinical manifestations and related symptoms of the patients disappeared significantly, there were no limb numbness, sensory disturbance and motor disturbance, the nerve conduction velocity increased by at least 5 meters per second, and the tendon reaction completely returned to normal, which was evaluated as effective ${ }^{[8]}$. If most of the symptoms are improved after treatment, and the tendon reaction is significantly better than before, but the nerve conduction examination shows that the lifting range is less than $5 \mathrm{~m} / \mathrm{s}$, which is evaluated as effective. However, if any of the above criteria cannot be met, or even the relevant symptoms continue to worsen, the evaluation is invalid.

\subsection{Statistical methods}

SPSS 20.0 is used for data processing. When $\mathrm{P}<0.05$, it is significant.

\section{Results}

Comparison of results found differences in effect. The total effective rate of the observation group was improved $(\mathrm{P}<0.05)$, which brought a more positive impact, and the clinical intervention effect was more satisfactory. The comparison of total effective rate between the two groups is shown in Table $\mathbf{1}$.

Table 1. Comparison of total effective rate between the two groups

\begin{tabular}{lccccc}
\hline Group & Number of cases & Remarkable effect & Effective & Inefficient & Total effective rate \\
\hline Observation group & 35 cases & 27 cases & 7 cases & 1 case & 34 cases \\
Control group & 35 cases & 21 cases & 6 cases & 8 cases & 27 cases \\
P & $/$ & & \multicolumn{2}{c}{$<0.05$} & \\
\hline
\end{tabular}




\section{Conclusion}

The long-term disorder of glycol-metabolism will further lead to the injury of peripheral nerves and the obstruction of blood and nutrient supply. Therefore, it has certain advantages to adopt a variety of methods for combined application in treatment ${ }^{[9]}$. From the perspective of traditional Chinese medicine, this problem is mainly due to the blood stasis caused by the problem of fin transport of the five cereals. Then, in the treatment, we should clarify the main purpose and the cause of this phenomenon, and finally take measures according to the situation of patients and adhere to the important principle of supplementing Qi and activating blood, which can achieve better results.

As mentioned above, for patients with peripheral neuropathy caused by glucose metabolism disorder, the treatment method of integrated traditional Chinese and western medicine is very helpful for the rapid recovery of the patient's health ${ }^{[10]}$. From the results, it also has a significant improvement compared with the original physical condition. Therefore, this method can achieve more satisfactory results.

\section{Disclosure statement}

The authors declare no conflict of interest.

\section{References}

[1] Zhao Y, Li Y, Guo Z, et al., 2019, Study on Treatment and Nursing of Senile Diabetic Peripheral Neuropathy with Integrated Traditional Chinese and Western Medicine. Diabetes World · Education (Early Issue), 016(009): 135-137.

[2] Yong L, Song BB, Jin H, et al., 2019, Clinical Effect of Integrated Traditional Chinese Medicine Therapy Based on the Theory of "Treating Flaccidity by Selecting the Yangming Meridian Alone" in Treatment of Restless Legs Syndrome During Peritoneal Dialysis. Journal of Anhui University of Chinese Medicine.

[3] Su Y, 2019, Analysis on the Clinical Effect of Integrated Traditional Chinese and Western Medicine in the Treatment of Severe Pneumonia Respiratory Failure. The Medical Forum.

[4] Zhao Z, Wang X, Wang S, et al., 2020, Study on the Clinical Assessment of Integrated Rehabilitation of Traditional Chinese Medicine and Western Medication for Acute Myocardial Infarction. Medicine, 99.

[5] Zhang W, 2020, Gastroenterology D O. Effect of Integrated Traditional Chinese and Western Medicine Treatment on Clinical Efficacy and Gastric Mucosa of Patients with Helicobacter Pylori Infected Gastric Ulcer. Medical Diet and Health.

[6] Feng X, Xie L, 2019, Meta-Analysis of Randomized Controlled Trials of Combined Treatment with DGSN and Western Medicine on Diabetic Peripheral Neuropathy. TMR Clinical Research, 002(001): 8-19.

[7] Fang XJ, Zheng GX, Huangfu YH, et al., 2020, Observation on Therapeutic Efficacy of Heat-Sensitive Moxibustion Plus Western Medicine for Diabetic Peripheral Neuropathy. Journal of Acupuncture and Tuina Science, 18(6):452-457.

[8] 2020, Traditional Chinese Medicine Foot Bath Combined with Acupoint Massage for the Treatment of Diabetic Peripheral Neuropathy: A Systematic Review and Meta - Analysis of 31 RCTs. Diabetes/Metabolism Research and Reviews, 36(2).

[9] Zhang S, Zhang S, Wang H, et al., 2019, Efficacy and Safety Evaluation of Integrated Traditional Chinese and Western Medicine in Treating Diabetic Peripheral Neuropathy with Qi Deficiency and Blood Stasis Syndrome. Pharmaceutical Care and Research, 019(004): 267-270.

[10] Shen Y, Wang S, Liu Y, et al., 2020, The Effects of Salvianolate Combined with Western Medicine on Diabetic Nephropathy: A Systematic Review and Meta-Analysis. Frontiers in Pharmacology, 11:851. 